\title{
Quantum versus classical instability of scalar fields in curved backgrounds
}

\author{
Raissa F. P. Mendes, ${ }^{1, *}$ George E. A. Matsas, ${ }^{1, \dagger}$ and Daniel A. T. Vanzella ${ }^{2,+}$ \\ ${ }^{1}$ Instituto de Física Teórica, Universidade Estadual Paulista, Rua Dr. Bento Teobaldo Ferraz 271, \\ 01140-070, São Paulo, São Paulo, Brazil \\ ${ }^{2}$ Instituto de Física de São Carlos, Universidade de São Paulo, Caixa Postal 369, \\ 13560-970, São Carlos, São Paulo, Brazil
}

(Received 7 October 2013; published 24 February 2014)

\begin{abstract}
General-relativistic stable spacetimes can be made unstable under the presence of certain nonminimally coupled free scalar fields. In this paper, we analyze the evolution of linear scalar-field perturbations in spherically symmetric spacetimes and compare the classical stability analysis with a recently discussed quantum field one. In particular, it is shown that vacuum fluctuations lead to natural seeds for the unstable phase, whereas in the classical framework the presence of such seeds in the initial conditions must be assumed.
\end{abstract}

DOI: $10.1103 /$ PhysRevD.89.047503

PACS numbers: $04.62 .+\mathrm{v}$

\section{INTRODUCTION}

It was shown that certain well-behaved spacetimes can induce an exponential growth of the vacuum energy density of some nonminimally coupled free scalar fields [1]. Particular astrophysically inspired realizations of this mechanism were explored in Refs. [2,3]. As the instability sets in, the system is driven to a new equilibrium state, generically inducing a burst of free scalar particles [4]. This quantum field effect has a classical counterpart, as remarked in Ref. [5]. There, the authors discuss the end state of the (classical) instability, and provide evidence that, for a certain range of field-to-curvature couplings, the system evolves to a "scalarized" final configuration (see also Refs. [6,7]).

In this paper we provide a more rigorous formulation of the relationship between the quantum and classical descriptions of the instability. For this purpose, we analyze the evolution of classical perturbations in a regular spherically symmetric spacetime within a quasinormal mode formalism based on the Laplace transform approach. This approach, which is often used to analyze the evolution of stable perturbations [8-10] is adapted here to unstable ones. We then show the similarities and differences between the quantum and classical descriptions and how quantum fluctuations can be simulated by classical perturbations of a given "small" amplitude. We set $c=G=1$.

\section{QUANTUM APPROACH TO INSTABILITY- A BRIEF REVIEW}

Let us assume a real massless free scalar field $\Phi$, on a spacetime $\left(\mathbb{R}^{4}, g_{a b}\right)$, governed by the field equation

$$
\left(-\nabla^{a} \nabla_{a}+\xi R\right) \Phi=0,
$$

\footnotetext{
rfpm@ift.unesp.br

matsas@ift.unesp.br

¥vanzella@ifsc.usp.br
}

where $R$ is the scalar curvature and $\xi \in \mathbb{R}$. Throughout this paper we restrict our attention to asymptotically flat, spherically symmetric spacetimes possessing no event horizons or singularities. Moreover, let the spacetime be Minkowski-like in the past, $\left(\mathbb{R}^{4}, \eta_{a b}\right)$, and static in the future, $\left(\mathbb{R}^{4}, g_{a b}\right)$, with $g_{a b}$ such that

$$
d s^{2}=-e^{2 \Xi(r)} d t^{2}+e^{2 \Lambda(r)} d r^{2}+r^{2}\left(d \theta^{2}+\sin ^{2} \theta d \phi^{2}\right),
$$

where $\Xi(r), \Lambda(r) \stackrel{r \sim 0}{\sim} r^{2}+\mathcal{O}\left(r^{4}\right)$ are bounded continuous functions with $\Xi(r), \Lambda(r) \stackrel{r \rightarrow \infty}{\rightarrow} 0$.

References [1-4] analyzed the case of a quantum field $\hat{\Phi}$, which satisfies Eq. (1), being stable on $\left(\mathbb{R}^{4}, \eta_{a b}\right)$ but unstable on $\left(\mathbb{R}^{4}, g_{a b}\right)$. In this setting, if the field is in the no-particle state $\left|0_{\text {in }}\right\rangle$ as described by static observers in $\left(\mathbb{R}^{4}, \eta_{a b}\right)$, then its vacuum fluctuations suffer an exponential amplification in time during the unstable phase:

$$
\left\langle 0_{\text {in }}\left|\hat{\Phi}^{2}\right| 0_{\text {in }}\right\rangle \sim \frac{\hbar \kappa e^{2 \bar{\Omega} t}}{8 \pi \bar{\Omega}}\left(\frac{\psi_{\bar{\Omega} 0}(r)}{r}\right)^{2}\left[1+\mathcal{O}\left(e^{-\epsilon t}\right)\right] .
$$

Here, $\psi_{\Omega l}(r)$, with $\Omega>0$ and $l \in \mathbb{N}$, obeys

$$
\left[-d^{2} / d x^{2}+V_{\mathrm{eff}}^{(l)}(r)\right] \psi_{\Omega l}[r(x)]=-\Omega^{2} \psi_{\Omega l}[r(x)],
$$

where the effective potential is given by

$$
V_{\mathrm{eff}}^{(l)}(r)=e^{2 \Xi}\left(\xi R+\frac{l(l+1)}{r^{2}}\right)+\frac{e^{2(\Xi-\Lambda)}}{r}\left(\frac{d \Xi}{d r}-\frac{d \Lambda}{d r}\right)
$$

and $x \in[0,+\infty)$ is defined as

$$
x(r) \equiv \int_{0}^{r} e^{\Lambda\left(r^{\prime}\right)-\Xi\left(r^{\prime}\right)} d r^{\prime} .
$$

Moreover, proper behavior of the field at the origin and infinity demands 


$$
\psi_{\Omega l}[r(x)]_{x=0}=0,\left.\quad \psi_{\Omega l}[r(x)]\right|_{x \rightarrow+\infty} \sim e^{-\Omega x},
$$

while normalization requires

$$
\int_{0}^{+\infty} d x \psi_{\Omega l}^{*} \psi_{\Omega^{\prime} l}=\delta_{\Omega \Omega^{\prime}}
$$

In Eq. (3), $\epsilon$ is some positive constant, $-\bar{\Omega}^{2}$ is the lowest negative eigenvalue of $-d^{2} / d x^{2}+V_{\text {eff }}^{(0)}(x)$, and $\kappa=$ const $\sim 1$ depends on the transition details to the unstable phase.

For a minimally coupled field $(\xi=0)$, it is possible to show that the operator $-d^{2} / d x^{2}+V_{\text {eff }}^{(l)}(x)$ has a purely positive spectrum and thus no solutions of Eq. (4) satisfying Eq. (7) exist (see the Appendix). However, for nonminimally coupled fields, the effective potential can be made sufficiently negative to allow the same operator to possess an additional negative (discrete) spectrum. This is the hallmark of the instability. See Refs. [1-4] for a complete discussion on the "vacuum awakening effect" (and Ref. [11] for a rigorous discussion on the quantization of unstable linear fields in globally static spacetimes).

\section{CONNECTION WITH CLASSICAL APPROACH TO INSTABILITY}

We now investigate the classical counterpart of the quantum instability described above. Consider the action $S_{\Psi}$ describing some matter field $\Psi$ defined on a spacetime ruled by the Einstein-Hilbert action $S_{\mathrm{EH}}$. Variation of $S_{\mathrm{EH}}+S_{\Psi}$ with respect to the metric gives

$$
G_{a b}=8 \pi T_{a b}^{\Psi},
$$

where $T_{a b}^{\Psi}=-(2 / \sqrt{-g}) \delta S_{\Psi} / \delta g^{a b}$ and $G_{a b}$ is the Einstein tensor. The line element (2) is assumed to be a solution of Eq. (9) for some matter distribution.

Next, let us perturb the system by introducing a free scalar field $\Phi$ ruled by Eq. (1). Then, Eq. (9) becomes

$$
G_{a b}=8 \pi\left(T_{a b}^{\Psi}+T_{a b}^{\Phi}\right)
$$

where

$$
\begin{aligned}
T_{a b}^{\Phi}= & (1-2 \xi) \nabla_{a} \Phi \nabla_{b} \Phi+\xi \Phi^{2} R_{a b}-2 \xi \Phi \nabla_{a} \nabla_{b} \Phi \\
& +(2 \xi-1 / 2)\left[\nabla_{c} \Phi \nabla^{c} \Phi+\xi R \Phi^{2}\right] g_{a b} .
\end{aligned}
$$

Inspired by the previous section where the quantum field was chosen to be in a suitable vacuum state, we aim to solve the classical field equations up to linear-order perturbation over the null-scalar-field configuration. Then, let us define $g_{a b} \equiv g_{a b}^{(0)}+g_{a b}^{(1)}, \Phi \equiv \Phi_{(0)}+\Phi_{(1)}$, where $\Phi_{(0)}=0$ and $\Phi_{(1)}$ is small in the sense that it engenders a small perturbation $g_{a b}^{(1)}$ with respect to the unperturbed background metric $g_{a b}^{(0)}$ given by Eq. (2). Because $T_{a b}^{\Phi}$ has a quadratic dependence in $\Phi$, we conclude that at first-order perturbation $g_{a b}=g_{a b}^{(0)}$ is a solution of Eq. (10), while $\Phi_{(1)}$ evolves according to

$$
\left(-\nabla^{a} \nabla_{a}+\xi R\right) \Phi_{(1)}=0
$$

on the fixed background $\left(\mathbb{R}^{4}, g_{a b}^{(0)}\right)$.

In contrast to the quantum case where vacuum fluctuations automatically trigger the exponential growth of $\left\langle 0_{\text {in }}\left|\hat{\Phi}^{2}\right| 0_{\text {in }}\right\rangle$ while keeping $\left\langle 0_{\text {in }}|\hat{\Phi}| 0_{\text {in }}\right\rangle=0$, in the classical context we shall postulate that at some instant, say $t=0$, some external agent drives $\Phi(t, \mathbf{x})$ out of its initial equilibrium state such that $\Phi(t, \mathbf{x}) \neq 0$ itself for $t>0$. Due to the spherical symmetry of the background spacetime (2), we decompose $\Phi_{(1)}$ as

$$
\Phi_{(1)}(t, r, \theta, \phi)=\sum_{l=0}^{\infty} \sum_{\mu=-l}^{l} \frac{\chi_{l \mu}(t, r)}{r} Y_{l \mu}(\theta, \phi),
$$

where the initial conditions are defined by specifying $\chi_{l \mu}(t, r)$ and $\partial_{t} \chi_{l \mu}(t, r)$ at $t=0$. In order to handle the initial conditions and establish a clear connection between the quantum analysis and the one using quasinormal modes, it will prove convenient to adapt the Laplace transform approach [9] to our case.

Let us define the Laplace transform of $\chi_{l \mu}(t, r)$ with respect to the time coordinate as

$$
\tilde{\chi}_{l \mu}(s, r) \equiv \int_{0}^{+\infty} e^{-s t} \chi_{l \mu}(t, r) d t, \quad s \in \mathbb{C},
$$

in some domain $\Re(s)>\gamma$ where $\tilde{\chi}_{l \mu}(s, r)$ is analytic. Here $\gamma$ is chosen so that $\left|\chi_{l \mu}(t, r)\right| \leq M e^{\gamma t}$ whenever $t>t_{0}$, for some $t_{0}, M \in \mathbb{R}^{+}$. From Eqs. (12)-(14), it follows that $\tilde{\chi}_{l \mu}(s, r)$ obeys

$$
-\partial_{x}^{2} \tilde{\chi}_{l \mu}(s, r)+\left(s^{2}+V_{\mathrm{eff}}^{(l)}\right) \tilde{\chi}_{l \mu}(s, r)=\mathcal{I}_{l \mu}(s, r),
$$

where

$$
\mathcal{I}_{l \mu}(s, r) \equiv\left[s \chi_{l \mu}(t, r)+\partial_{t} \chi_{l \mu}(t, r)\right]_{t=0}
$$

is fixed by the initial conditions. Inspired by the quantum case where the instability is triggered by vacuum fluctuations which drop fast at infinity [see Eq. (3) with $\psi_{\bar{\Omega} 0}$ obeying Eq. (7)], we consider here that the system is perturbed by a classical seed localized in space. Thus, we assume that $\chi_{l \mu}(t, r)$ and $\partial_{t} \chi_{l_{\mu}}(t, r)$ have compact support as functions of $r$ at $t=0$ in which case $\mathcal{I}_{l \mu}(s, r)=0$ for $r>\ell=$ const.

As a consequence of our localized initial condition assumption, we have for large enough $r$ that

$$
\left|\tilde{\chi}_{l \mu}(s, r)\right| \leq \int_{x(r)-x(\ell)}^{+\infty} M e^{-(\Re(s)-\gamma) t} d t,
$$


where we have used Eq. (14) and the causal propagation property of Eq. (12). Hence, after performing the integration in Eq. (17), we conclude that

$$
\lim _{r \rightarrow+\infty} \tilde{\chi}_{l \mu}(s, r)=0 .
$$

The detailed form of $\tilde{\chi}_{l \mu}(s, r)$ will depend on Eq. (15). A general solution of Eq. (15) can be cast as

$$
\tilde{\chi}_{l \mu}(s, r)=\int_{0}^{+\infty} G_{l}\left(s ; r, r^{\prime}\right) \mathcal{I}_{l \mu}\left(s, r^{\prime}\right) d x^{\prime},
$$

where $r^{\prime} \equiv r\left(x^{\prime}\right)$ and $G_{l}\left(s ; r, r^{\prime}\right)$ satisfies

$$
-\partial_{x}^{2} G_{l}\left(s ; r, r^{\prime}\right)+\left(s^{2}+V_{\mathrm{eff}}^{(l)}\right) G_{l}\left(s ; r, r^{\prime}\right)=\delta\left(x-x^{\prime}\right) .
$$

Any solution of Eq. (20) can be written as

$$
G_{l}\left(s ; r, r^{\prime}\right)=f_{l}^{-}\left(s, r_{<}\right) f_{l}^{+}\left(s, r_{>}\right) / W_{l}(s),
$$

where $f_{l}^{ \pm}(s, r)$ are two linearly independent solutions of the homogeneous equation

$$
-\partial_{x}^{2} f_{l}^{ \pm}(s, r)+\left(s^{2}+V_{\mathrm{eff}}^{(l)}\right) f_{l}^{ \pm}(s, r)=0
$$

with $r_{<} \equiv \min \left(r, r^{\prime}\right)$ and $r_{>} \equiv \max \left(r, r^{\prime}\right)$. Here,

$$
W_{l}(s) \equiv f_{l}^{+}(s, r) \partial_{x} f_{l}^{-}(s, r)-f_{l}^{-}(s, r) \partial_{x} f_{l}^{+}(s, r) .
$$

We note that $G_{l}\left(s ; r, r^{\prime}\right)$ is not affected by rescaling $f_{l}^{ \pm}(s, r)$ through any (nonzero) multiplicative constant.

The Green function $G_{l}\left(s ; r, r^{\prime}\right)$ is completely specified by Eq. (21) by imposing proper boundary conditions to $f_{l}^{ \pm}(s, r)$. Equation (18) combined with Eqs. (19) and (21) leads to

$$
\left.f_{l}^{+}(s, r)\right|_{r \rightarrow+\infty} \sim e^{-s x}
$$

for $\gamma \geq 0$. In addition, the regularity condition imposed to $\Phi_{(1)}$ at the origin demands $\left.\chi_{l \mu}(t, r)\right|_{r=0}=0$ and, thus,

$$
\left.f_{l}^{-}(s, r)\right|_{r=0}=0 .
$$

Note that for large enough $|s|$,

$$
G_{l}\left(s ; r, r^{\prime}\right) \sim e^{-s x_{>}} \sinh \left(s x_{<}\right) / s .
$$

Eventually, $\chi_{l \mu}(t, r)$ is recovered through the inverse Laplace transform

$$
\chi_{l \mu}(t, r)=\frac{1}{2 \pi i} \int_{\kappa-i \infty}^{\kappa+i \infty} e^{s t} \tilde{\chi}_{l \mu}(s, r) d s,
$$

where $\kappa>\gamma$, and $\tilde{\chi}_{l \mu}(s, r)$ is given in Eq. (19). Then, $\Phi_{(1)}$ is straightforwardly obtained from Eq. (13).
For $t \geq 0$ it is convenient to extend $\tilde{\chi}_{l \mu}(s, r)$ to $\Re(s) \leq \gamma$ in order to use the residue theorem to calculate Eq. (26). The extension of $\tilde{\chi}_{l \mu}(s, r)$ to the remaining complex plane raises poles including the ones which codify the instabilities in which we are interested. The singularities of $\tilde{\chi}_{l \mu}(s, r)$ in the region $\Re(s) \leq \gamma$ come from the Green function $G_{l}\left(s ; r, r^{\prime}\right)$, since $\mathcal{I}_{l \mu}(s, r)$ is an entire function of $s \in \mathbb{C}$ [see Eq. (19)]. These, in turn, can be traced back either to singularities of $f_{l}^{ \pm}(s, r)$ or to zeros of the Wronskian [see Eq. (21)]. The former will depend on global properties of the effective potential [12]. For $V_{\text {eff }}^{(l)}$ associated with compact objects, $f_{l}^{+}(s, r)$ will possess a logarithmic singularity at $s=0$ [13]. Moreover, the zeros of the Wronskian, $W_{l}\left(s_{0}\right)=0$, will give rise to simple poles of $\tilde{\chi}_{l \mu}(s, r)$ at $s=s_{0}$ provided that $d W_{l}(s) /\left.d s\right|_{s=s_{0}} \neq$ 0 [see below Eq. (32)]. Then, $f_{l}^{-}\left(s_{0}, r\right)$ and $f_{l}^{+}\left(s_{0}, r\right)$ are linearly dependent functions and can be assumed to be equal with no loss of generality: $f_{l}^{ \pm}\left(s_{0}, r\right) \equiv f_{l}\left(s_{0}, r\right)$. Figure 1 illustrates the singularity pattern of $\tilde{\chi}_{l \mu}(s, r)$.

We calculate Eq. (26) for $t>0$ through the residue theorem with integration contour shown in Fig. 1:

$$
\begin{aligned}
\int_{\kappa-i \infty}^{\kappa+i \infty} e^{s t} \tilde{\chi}_{l \mu}(s, r) d s= & 2 \pi i \sum_{\text {poles }} \operatorname{Res}\left[e^{s t} \tilde{\chi}_{l \mu}(s, r)\right] \\
& -\int_{L_{1} \Gamma_{1} L \Gamma_{2} L_{2}} e^{s t} \tilde{\chi}_{l \mu}(s, r) d s .
\end{aligned}
$$

The $\Gamma_{1}, \Gamma_{2}$, and $L$ contributions are well studied in the literature [14] (see also, e.g., Refs. [8,15]). The $L_{1}, L_{2}$ contributions which appear as a consequence of the existence of poles with $\Re\left(s_{0}\right)>0$ can be seen to vanish by inserting Eqs. (16) and (25) in Eq. (19) and noting that we end up with a sum of two integrals corresponding to both terms of Eq. (16). For $T \equiv|\Im(s)| \rightarrow+\infty$, one of them

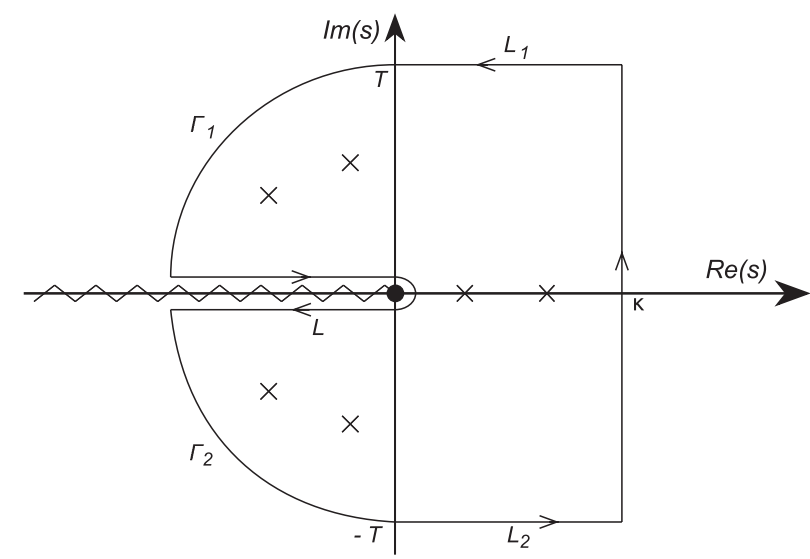

FIG. 1. The singularity structure of $\tilde{\chi}_{l \mu}(s, r)$ in the plane $s \in \mathbb{C}$ and the integration contour chosen to calculate $\chi_{\iota_{\mu}}(t, r)(t>0)$ are exhibited. The logarithmic singularity is at the origin and the corresponding branch cut is set on the negative real axis. The poles of $\tilde{\chi}_{l \mu}(s, r)$ are represented by $\times$ symbols. We focus on those with $s_{0}>0$ which are associated to instability. 
goes to zero as $1 / T$ while the other one vanishes as a result of the rapid oscillation of the integrand in this limit.

Then, by using Eq. (27) in Eq. (26) we have

$$
\chi_{l \mu}(t, r)=\sum_{\text {poles }} c_{l \mu}\left(s_{0}\right) e^{s_{0} t} f_{l}\left(s_{0}, r\right)+\text { contour terms }
$$

where

$$
c_{l \mu}\left(s_{0}\right) \equiv \frac{1}{d W_{l} /\left.d s\right|_{s=s_{0}}} \int_{0}^{+\infty} f_{l}\left(s_{0}, r^{\prime}\right) \mathcal{I}_{l \mu}\left(s_{0}, r^{\prime}\right) d x^{\prime} .
$$

Poles with $\Re\left(s_{0}\right)<0$ will correspond to exponentially damped oscillating-in-time quasinormal modes [10]. (Despite the asymptotic behavior exhibited by $f_{l}^{+}\left(s_{0}, r\right)$ in Eq. (23), for $\Re\left(s_{0}\right)<0, \chi_{l \mu}(t, r)$ will be well behaved at infinity due to the compact support initial condition assumption [16].) Here, we focus on poles with $\Re\left(s_{0}\right)>$ 0 which will drive $\chi_{l \mu}(t, r)$ to grow exponentially in time. The radial part of $\chi_{l \mu}(t, r)$ is determined by $f_{l}\left(s_{0}, r\right)$ as given by Eq. (28) and satisfies Eq. (22) with boundary conditions (23) and (24). Such a solution is a normalized eigenvector of the Hermitian operator $-d^{2} / d x^{2}+V_{\mathrm{eff}}^{(l)}(x)$, from which we conclude that unstable quasinormal modes have $\mathfrak{s}\left(s_{0}\right)=0$ (see Fig. 1). Let us denote by $\bar{s}_{0}$ the pole with largest positive $\mathfrak{R}\left(s_{0}\right)$ among them. Then, as far as our first order perturbation is valid, the "late-time" behavior of $\chi_{l \mu}(t, r)$ can be cast in the form

$$
\chi_{l \mu}(t, r) \sim c_{l \mu}\left(\bar{s}_{0}\right) e^{\bar{s}_{0} t} f_{l}\left(\bar{s}_{0}, r\right)\left[1+\mathcal{O}\left(e^{-\epsilon t}\right)\right],
$$

where $\epsilon$ is some positive constant. Inserting this expression in Eq. (13) and noting that the dominant contribution comes from $l=0$, we obtain

$$
\begin{aligned}
\Phi_{(1)}^{2} \sim & {\left[\int_{0}^{+\infty} f_{0}\left(\bar{s}_{0}, r^{\prime}\right) \mathcal{I}_{00}\left(\bar{s}_{0}, r^{\prime}\right) d x^{\prime}\right]^{2} \frac{e^{2 \bar{s}_{0} t}}{16 \pi \bar{s}_{0}^{2}}\left[\frac{f_{0}\left(\bar{s}_{0}, r\right)}{r}\right]^{2} } \\
& \times\left[1+\mathcal{O}\left(e^{-\epsilon t}\right)\right],
\end{aligned}
$$

where we have used $d W_{l} /\left.d s\right|_{s=\bar{s}_{0}}=-2 \bar{s}_{0}$ and that $f_{l}^{ \pm}(s, r)$ can be rescaled arbitrarily to demand

$$
\int_{0}^{+\infty} f_{l}^{2}\left(\bar{s}_{0}, r\right) d x=1
$$

The expression for the Wronskian derivative can be obtained by adapting a derivation in [17] to real positive poles: first, we use Eqs. (22) and (32) to write

$$
\begin{aligned}
1 & =\lim _{s \rightarrow \bar{s}_{0}} \int_{0}^{X \rightarrow \infty} f_{l}^{-}\left(\bar{s}_{0}, r\right) f_{l}^{+}(s, r) d x \\
& =\lim _{s \rightarrow \bar{s}_{0}} \frac{\left[f_{l}^{+}(s, r) \partial_{x} f_{l}^{-}\left(\bar{s}_{0}, r\right)-f_{l}^{-}\left(\bar{s}_{0}, r\right) \partial_{x} f_{l}^{+}(s, r)\right]_{r=0}}{s^{2}-\bar{s}_{0}^{2}},
\end{aligned}
$$

where the superior integration limit term vanishes as can be seen by using $\partial_{x} f_{l}^{+}(s, r) \approx-s f_{l}^{+}(s, r)$ for $X$ large enough [see Eq. (23)] and the fact that $f_{l}\left(\bar{s}_{0}, r\right)=$ $f_{l}^{ \pm}\left(\bar{s}_{0}, r\right) \stackrel{X \rightarrow+\infty}{\longrightarrow} 0$. Then, by making the change $\bar{s}_{0} \rightarrow s$ in the numerator of Eq. (33) to identify it with the Wronskian and using the L'Hospital rule, we obtain $d W_{l} /\left.d s\right|_{s=\bar{s}_{0}}=-2 \bar{s}_{0}$.

In order to compare the quantum and classical observables $\left\langle 0_{\text {in }}\left|\hat{\Phi}^{2}\right| 0_{\text {in }}\right\rangle$ and $\Phi_{(1)}^{2}$ given by Eqs. (3) and (31), respectively, we identify $\psi_{\bar{\Omega} 0}(r)$ and $f_{0}\left(\bar{s}_{0}, r\right)$ for $\bar{\Omega}=\bar{s}_{0}$, since they satisfy the same differential equation [see Eqs. (4) and (22)] with identical boundary conditions and compatible normalizations.

Next, note that $\left\langle 0_{\text {in }}\left|\hat{\Phi}^{2}\right| 0_{\text {in }}\right\rangle$ and $\Phi_{(1)}^{2}$ only differ by a multiplicative factor which includes $\mathcal{I}_{00}\left(\bar{s}_{0}, r\right)$. This is natural since in the classical context the evolution of the scalar field depends on the choice of the initial conditions, while in the quantum case the instability is triggered by vacuum fluctuations encoded on the choice of the quantum state. Now, let us suppose a compact star with radius $r=R_{s}$ and choose a typical initial condition as, e.g., $\left.\chi_{00}(t, r)\right|_{t=0}=A \Theta\left(R_{s}-r\right)$, $\left.\partial_{t} \chi_{00}(t, r)\right|_{t=0}=0$. In this case,

$$
\frac{\Phi_{(1)}^{2}}{\left\langle 0_{\text {in }}\left|\hat{\Phi}^{2}\right| 0_{\text {in }}\right\rangle}=\frac{A^{2} \bar{s}_{0}}{2 \hbar \kappa}\left[\int_{0}^{x\left(R_{s}\right)} f_{0}\left(\bar{s}_{0}, r^{\prime}\right) d x^{\prime}\right]^{2} .
$$

Finally, by using (i) Eq. (32), (ii) the fact that $f_{0}\left(\bar{s}_{0}, r\right)$ decreases fast for $r \gg R_{s}$, implying $f_{0}\left(\bar{s}_{0}, r\right) \sim 1 / R_{s}^{1 / 2}$, and (iii) $R_{s}^{-2} \sim\left|V_{\text {eff }}^{(l)}\right| \sim \bar{s}_{0}^{2}$, we cast Eq. (34) as

$$
\Phi_{(1)}^{2} /\left\langle 0_{\text {in }}\left|\hat{\Phi}^{2}\right| 0_{\text {in }}\right\rangle \sim A^{2} / 2 \hbar .
$$

The consequence of condition (ii) used above, namely $f_{0}\left(\bar{s}_{0}, r\right) \sim 1 / R_{s}^{1 / 2}$, comes by noting that $f_{0}\left(\bar{s}_{0}, r\right)$ gives a negligible contribution in Eq. (32) for $r \gtrsim R_{s} \sim x\left(R_{s}\right)$. Condition (iii) comes from Eq. (22) by demanding that $\left|V_{\text {eff }}^{(l)}\right|$ be at least of order $R_{s}^{-2}$ to make the potential "deep" enough to allow bound solutions [see Eq. (32)]. Conversely, by assuming the existence of bound solutions the corresponding $\bar{s}_{0}^{2}$ is typically of the order of $\left|V_{\text {eff }}^{(l)}\right|$.

\section{FINAL DISCUSSIONS}

We have shown how $\hbar$ can be made to appear in $\Phi_{(1)}$ by properly choosing the magnitude of the initial amplitude as $|A| \sim \hbar^{1 / 2}$. However, in this case a quantum mechanical treatment should be more suitable. As long as fluctuations of the stress-energy-momentum tensor are "reasonably" small [18], the spacetime will respond according to the semiclassical Einstein equations $G_{a b}=8 \pi\left\langle 0_{\text {in }}\left|\hat{T}_{a b}\right| 0_{\text {in }}\right\rangle$. The corresponding evolution is a highly nontrivial task. However, for unstable systems, it seems reasonable that when vacuum fluctuations become large enough they 
should somehow "collapse" into classical perturbations (see, e.g., [19]) in a process analogous to the formation of the cosmic-microwave-background anisotropies from primordial vacuum fluctuations. Afterwards, the system should be properly evolved through the classical equations of motion (see, e.g., [7,20]).

\section{ACKNOWLEDGMENTS}

We are thankful to Bob Wald for comments in the 19th International Conference on General Relativity and Gravitation and in the 12th Midwest Relativity Meeting which partially motivated this paper. We also thank A. Landulfo and P. Pani for carefully reading the manuscript. R. M. was supported by the São Paulo Research Foundation (FAPESP) under Grant No. 2011/06429-3. G. M. and D. V. acknowledge partial support from FAPESP under Grant No. 2007/55449-1. G. M. also acknowledges Conselho Nacional de Desenvolvimento Científico e Tecnológico (CNPq) for partial support.

\section{APPENDIX}

Here, we show that Eq. (4) with $\xi=0$ has no solutions satisfying conditions (7). It suffices to analyze the $l=0$ case because by vanishing the $l(l+1) / r^{2}$ positive term in the effective potential (5), we improve our chances of finding bound solutions by "deepening" $V_{\mathrm{eff}}^{(l)}$. Thus, we seek normalizable solutions of

$$
-d^{2} \psi_{\Omega 0} / d x^{2}+\left.V_{\mathrm{eff}}^{(0)}\right|_{\xi=0} \psi_{\Omega 0}=-\Omega^{2} \psi_{\Omega 0},
$$

where $\left.V_{\mathrm{eff}}^{(0)}\right|_{\xi=0}=\left(e^{2(\Xi-\Lambda)} / r\right)(d \Xi / d r-d \Lambda / d r)$ with

$$
\left.\psi_{\Omega 0}\right|_{x=0}=0
$$

demanded by field regularity [see Eq. (7)]. Furthermore, the fact that $\left.V_{\text {eff }}^{(0)}\right|_{\xi=0}$ is nonsingular at the origin demands $d \psi_{\Omega 0} /\left.d x\right|_{x=0}=$ const $\neq 0$. For convenience, we choose

$$
d \psi_{\Omega 0} /\left.d x\right|_{x=0}=\left.C \exp [\Xi-\Lambda]\right|_{x=0}
$$

with $C$ being a nonvanishing constant which is fixed by Eq. (8). We recall that Eqs. (A2)-(A3) uniquely determine the solutions of Eq. (A1).

On the other hand, we see from Eq. (6) that $f(x) \equiv$ $\operatorname{Cr}(x)$ satisfies (i) the same differential equation as Eq. (A1) provided that $\Omega=0:-d^{2} f / d x^{2}+\left.V_{\text {eff }}^{(0)}\right|_{\xi=0} f=0$, and (ii) conditions similar to Eqs. (A2) and (A3), i.e., $\left.f(x)\right|_{x=0}=0$, and $d f /\left.d x\right|_{x=0}=\left.C \exp [\Xi-\Lambda]\right|_{x=0}$. Now, because $f(x)=\operatorname{Cr}(x)$ is a monotonically increasing function of $x$ [see Eq. (6)], we immediately conclude that Eq. (A1) with $\Omega=0$ does not possess solutions satisfying Eq. (7). Then, because $\Omega^{2}$ in Eq. (A1) just increases $d^{2} \psi_{\Omega 0} / d x^{2}$, we also get that the same conclusion is valid when $\Omega$ is nonzero. This implies that there are no unstable modes for minimally coupled scalar fields in asymptotically flat spherically symmetric static spacetimes containing no event horizons or singularities, which is compatible with all known literature. Although the derivation above assumed a massless field, the same conclusion holds for massive ones, $m \neq 0$, since in this case the effective potential is altered by the addition of a positive term, $m^{2} e^{2 \Xi}$, which "shallows" $\left.V_{\mathrm{eff}}^{(l)}\right|_{\xi=0}$ even more.
[1] W. C. C. Lima and D. A. T. Vanzella, Phys. Rev. Lett. 104, 161102 (2010).

[2] W. C. C. Lima, G. E. A. Matsas, and D. A. T. Vanzella, Phys. Rev. Lett. 105, 151102 (2010).

[3] W. C. C. Lima, R. F. P. Mendes, G. E. A. Matsas, and D. A. T. Vanzella, Phys. Rev. D 87, 104039 (2013).

[4] A. G. S. Landulfo, W. C. C. Lima, G. E. A. Matsas, and D. A. T. Vanzella, Phys. Rev. D 86, 104025 (2012).

[5] P. Pani, V. Cardoso, E. Berti, J. Read, and M. Salgado, Phys. Rev. D 83, 081501 (2011).

[6] T. Harada, Prog. Theor. Phys. 98, 359 (1997).

[7] J. Novak, Phys. Rev. D 58, 064019 (1998).

[8] E. W. Leaver, Phys. Rev. D 34, 384 (1986).

[9] H.-P. Nollert and B. G. Schmidt, Phys. Rev. D 45, 2617 (1992).

[10] H.-P. Nollert, Classical Quantum Gravity 16, R159 (1999); K. D. Kokkotas and B. G. Schmidt, Living Rev. Relativity 2, 2 (1999).

[11] W. C. C. Lima, Phys. Rev. D 88, 124005 (2013); B. Schroer and J. A. Swieca, Phys. Rev. D 2, 2938 (1970); B. Schroer, Phys. Rev. D 3, 1764 (1971).
[12] R. G. Newton, J. Math. Phys. (N.Y.) 1, 319 (1960); Scattering Theory of Waves and Particles (Springer-Verlag, Berlin, 1982).

[13] E. S. C. Ching, P. T. Leung, W. M. Suen, and K. Young, Phys. Rev. D 52, 2118 (1995).

[14] Briefly, the integration on $\Gamma_{1}, \Gamma_{2}$ contributes to the early signal (direct propagation) and vanishes after a certain time interval, while the one along $L$ captures the tail of the late-time signal (in the absence of instabilities).

[15] N. Andersson, Phys. Rev. D 55, 468 (1997).

[16] B. S. Kay and R. M. Wald, Classical Quantum Gravity 4, 893 (1987).

[17] E. S. C. Ching, P. T. Leung, W. M. Suen, and K. Young, Phys. Rev. Lett. 74, 4588 (1995).

[18] C. Kuo and L.H. Ford, Phys. Rev. D 47, 4510 (1993).

[19] M. P. Blencowe, Phys. Rev. Lett. 111, 021302 (2013).

[20] M. Ruiz, J. C. Degollado, M. Alcubierre, D. Núñez, and M. Salgado, Phys. Rev. D 86, 104044 (2012). 